\title{
FOXG1 syndrome: genotype-phenotype association in 83 patients with FOXG1 variants
}

Diana Mitter, MD ${ }^{1}$, Milka Pringsheim, $\mathrm{MD}^{2,3}$, Marc Kaulisch, Dipl. Pol ${ }^{4}, \mathrm{Kim}^{5}$ Sarah Plümacher ${ }^{5}$, Simone Schröder, PhD ${ }^{5}$, Rita Warthemann, PhD $^{5}$, Rami Abou Jamra, MD ${ }^{1}$, Martina Baethmann, MD ${ }^{6}$, Thomas Bast, $\mathrm{MD}^{7}$, Hans-Martin Büttel, $\mathrm{MD}^{8}$, Julie S. Cohen, $\mathrm{ScM}^{9}$, Elizabeth Conover, MS, MSN ${ }^{10}$, Carolina Courage, MD ${ }^{11}$, Angelika Eger, MD ${ }^{12}$, Ali Fatemi, MD, PhD ${ }^{9}$, Theresa A. Grebe, MD ${ }^{13}$, Natalie S. Hauser, MD ${ }^{14}$, Wolfram Heinritz, MD ${ }^{15}$, Katherine L. Helbig, MS ${ }^{16}$, Marion Heruth, MD ${ }^{17}$, Dagmar Huhle, MD ${ }^{18}$, Karen Höft, MD ${ }^{19}$, Stephanie Karch, MD ${ }^{20}$, Gerhard Kluger, MD ${ }^{2,3}$,

G. Christoph Korenke ${ }^{21}$, Johannes R. Lemke, MD ${ }^{1}$, Richard E. Lutz, MD ${ }^{10}$, Steffi Patzer, MD ${ }^{22}$, Isabelle Prehl, Dipl. Biol ${ }^{23}$, Konstanze Hoertnagel, MD ${ }^{23}$, Keri Ramsey, BSN ${ }^{24}$, Tina Rating, MD ${ }^{25}$ Angelika Rieß, MD ${ }^{26}$, Luis Rohena, MD²7, Mareike Schimmel, MD ${ }^{28}$, Rachel Westman, MS, LCGC ${ }^{29}$, Frank-Martin Zech, MD ${ }^{30}$, Barbara Zoll, MD, PhD ${ }^{31}$, Dörthe Malzahn, $\mathrm{PhD}^{32}$, Birgit Zirn, MD, PhD ${ }^{33}$ and Knut Brockmann, $\mathrm{MD}^{34}$

Purpose: The study aimed at widening the clinical and genetic spectrum and assessing genotype-phenotype associations in FOXG1 syndrome due to FOXG1 variants.

Methods: We compiled 30 new and 53 reported patients with a heterozygous pathogenic or likely pathogenic variant in FOXG1. We grouped patients according to type and location of the variant. Statistical analysis of molecular and clinical data was performed using Fisher's exact test and a nonparametric multivariate test.

Results: Among the 30 new patients, we identified 19 novel FOXG1 variants. Among the total group of 83 patients, there were 54 variants: 20 frameshift (37\%), 17 missense (31\%), 15 nonsense (28\%), and 2 in-frame variants (4\%). Frameshift and nonsense variants are distributed over all FOXG1 protein domains; missense variants cluster within the conserved forkhead domain. We found a higher phenotypic variability than previously described. Genotypephenotype association revealed significant differences in psychomotor development and neurological features between FOXG1 genotype groups. More severe phenotypes were associated with truncating FOXG1 variants in the $\mathrm{N}$-terminal domain and the forkhead domain (except conserved site 1) and milder phenotypes with missense variants in the forkhead conserved site 1 .

Conclusions: These data may serve for improved interpretation of new FOXG1 sequence variants and well-founded genetic counseling.

Genet Med advance online publication 29 June 2017

Key Words: congenital variant of Rett syndrome; FOXG1 phenotypic spectrum; FOXG1 variants; genotype-phenotype association

\footnotetext{
${ }^{1}$ Institute of Human Genetics, University Hospital Leipzig, Leipzig, Germany; ${ }^{2}$ Klinik für Neuropädiatrie und Neurologische Rehabilitation, Epilepsiezentrum für Kinder und Jugendliche, Schön Klinik Vogtareuth, Vogtareuth, Germany; ${ }^{3}$ PMU Salzburg, Salzburg, Austria; ${ }^{4}$ Department für Forschungs- und Transferservice, Universität Leipzig, Leipzig, Germany; ${ }^{5}$ Klinik für Kinder- und Jugendmedizin, Universitätsmedizin Göttingen, Göttingen, Germany; ${ }^{6}$ Kinderklinik, Klinikum Dritter Orden, Munich, Germany; ${ }^{7}$ Epilepsiezentrum Kork, Kehl-Kork, Germany; ${ }^{8}$ Sozialpädiatrisches Zentrum, SLK-Kliniken Heilbronn, Heilbronn, Germany; ${ }^{9}$ Moser Center for Leukodystrophies and Neurogenetics Service, Kennedy Krieger Institute, Johns Hopkins Medical Institution, Baltimore, Maryland, USA; ${ }^{10}$ Department of Genetic Medicine, Munroe Meyer Institute, University of Nebraska Medical Center Omaha, Omaha, Nebraska, US; ${ }^{11}$ Division of Human Genetics, Department of Pediatrics, Inselspital, University of Bern, Bern, Switzerland; ${ }^{12}$ Sozialpädiatrisches Zentrum Leipzig (Frühe Hilfe Leipzig), Leipzig, Germany; ${ }^{13}$ Division of Genetics and Metabolism, Phoenix Children's Hospital, Phoenix, Arizona, USA; ${ }^{14}$ Department of Medical Genomics Inova Translational Medicine Institute, Inova Fairfax Hospital, Falls Church, Virginia, USA; ${ }^{15}$ Praxis für Humangenetik Cottbus, Cottbus, Germany; ${ }^{16}$ Division of Clinical Genomics, Ambry Genetics, Aliso Viejo, California, USA; ${ }^{17}$ Klinik für Kinder- und Jugendmedizin, Sana Kliniken Leipziger Land, Borna, Germany; ${ }^{18}$ Praxis für Humangenetik Leipzig, Leipzig, Germany; ${ }^{19}$ Klinik für Kinder- und Jugendmedizin, Klinikum Magdeburg, Magdeburg, Germany; ${ }^{20}$ Sozialpädiatrisches Zentrum, Klinik für Kinder- und Jugendmedizin, Universitätsklinikum Heidelberg, Heidelberg, Germany; ${ }^{21}$ Klinik für Neuropädiatrie und Angeborene Stoffwechselerkrankungen, Elisabeth Kinderkrankenhaus, Klinikum Oldenburg, Oldenburg, Germany; ${ }^{22}$ Klinik für Kinder- und Jugendmedizin, Krankenhaus St. Elisabeth und St. Barbara, Halle/Saale, Germany; ${ }^{23} \mathrm{CeGaT}$, Tübingen, Germany; ${ }^{24}$ Center For Rare Childhood Disorders, Translational Genomics Research Institute, Phoenix, Arizona, USA; ${ }^{25}$ Sozialpädiatrisches Institut, Klinikum Bremen-Mitte, Bremen-Mitte, Germany; ${ }^{26}$ Institut für Medizinische Genetik und Angewandte Genomik, Universitätsklinikum Tübingen, Tübingen, Germany; ${ }^{27}$ Division of Medical Genetics, Department of Pediatrics, San Antonio Military Medical Center, San Antonio, Texas, USA; ${ }^{28}$ Klinik für Kinder und Jugendliche, Klinikum Augsburg, Augsburg, Germany; ${ }^{29}$ Children's Specialty Center, St. Luke's Children's Hospital, Boise, Idaho, USA; ${ }^{30}$ Klinik für Kinder- und Jugendmedizin, St. Vincenz-Krankenhaus Paderborn, Paderborn, Germany; ${ }^{31}$ Institut für Humangenetik, Universitätsmedizin Göttingen, Göttingen, Germany; ${ }^{32}$ Department of Genetic Epidemiology, University Medical Center, Georg-August University Göttingen, Göttingen, Germany; ${ }^{33}$ Genetic Counselling and Diagnostic, Genetikum Stuttgart, Stuttgart, Germany; ${ }^{34}$ SPZ, Klinik für Kinder- und Jugendmedizin Universitätsmedizin Göttingen, Göttingen, Germany. Correspondence: Diana Mitter (diana.mitter@medizin.uni-leipzig.de)
} The last two authors contributed equally to this work. 


\section{ORIGINAL RESEARCH ARTICLE}

\section{INTRODUCTION}

FOXG1 syndrome (OMIM 613454) is a rare neurodevelopmental disorder associated with heterozygous variants in the forkhead box G1 (FOXG1) gene (OMIM 164874, accession number: P55316). These include FOXG1 variants and chromosomal microaberrations, namely deletions $s^{1-15}$ and

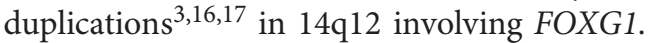

The phenotype in patients carrying a FOXG1 variant including FOXG1 deletions is designated "congenital variant

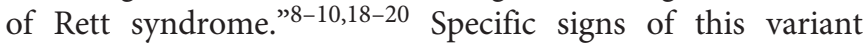
include the presence of a dyskinetic-hyperkinetic movement disorder, the lack of regression or respiratory arrhythmia, and the occurrence of cerebral malformations in patients with a FOXG1 variant. ${ }^{2}$ Main clinical features observed in association with FOXG1 variants comprise impairment of postnatal growth, primary (congenital) or secondary (postnatal) microcephaly, severe intellectual disability with absent speech development, epilepsy, stereotypies and dyskinesia, abnormal sleep patterns, unexplained episodes of crying, gastroesophageal reflux, and recurrent aspiration. ${ }^{2}$ Neuroimaging showed hypogenesis of corpus callosum, simplified gyral pattern, and reduced white matter volume in the frontal lobes and frontal pachygyria in a few cases. ${ }^{2}$ This recognizable clinical phenotype is also designated the FOXG1 syndrome, ${ }^{2}$ and in this article we use the term "FOXG1 syndrome" as equivalent to the original designation "congenital variant of Rett syndrome." However, the complex phenotypic spectrum is still expanding.

In this study, we analyzed data for 30 previously unreported patients and 53 patients described in the literature $(n=83$ patients in total) with a pathogenic or likely pathogenic FOXG1 variant according to the recommendations for interpretation of sequence variants published by the American College of Medical Genetics and Genomics (ACMG). ${ }^{21}$ We aimed at refining the phenotypic spectrum related to FOXG1 variants and at establishing further and more differentiated genotype-phenotype associations allowing for improved genetic counseling of affected families.

\section{MATERIALS AND METHODS}

In collaboration with pediatric neurologists and human geneticists from Germany, Switzerland, and the United States, we collected 30 new patients from 28 families with a FOXG1 variant. Standardized phenotypic data were collected by review of the clinical histories and follow-up investigations. Additional data were compiled in parental telephone interviews using a standardized questionnaire. All available cranial magnetic resonance image (MRI) data sets $(n=22)$ were reviewed by both neuroradiologists and neurologists. Based on a Medline search, we identified additional 53 patients from 48 families with a FOXG1 variant. ${ }^{1-3,7,9,10,14,18-20,22-26}$ All available clinical, neuroimaging, and molecular data were collected from these reports and assembled with the data for the 30 new patients (Table 1, Supplementary Table S1 online). Phenotyping had been blinded regarding retrospect assignment of FOXG1 genotype groups.
To comprehensively quantify the clinical phenotypes associated with pathogenic FOXG1 variants in all 83 patients, we defined a new FOXG1 severity score as the mean singleitem rating, obtained within an individual by averaging over 20 phenotypic items in five categories: somatic growth (4 items), motor and speech development (4 items, if applicable according to patient's age), behavior (3 items), neurological features (6 items), and MRI anomalies (3 items) (Supplementary Table S4). Phenotypic items were rated with 0 to 2 points, ensuring the same scale, with higher scores indicating a more severe clinical phenotype. The severity score averaged over available items for an individual and was calculated only for patients $(n=49)$ for whom there was at least one item in each of the five categories.

The score cannot reflect the full clinical variability of FOXG1 syndrome but rather aimed at comprising the most important domains of the clinical phenotype to provide a simple tool for quantification of the overall clinical impact of the FOXG1 variants. The score was assigned by three authors based on the clinical data provided by the referring specialists for the new patients and the data extracted from the previously published reports. Thus, criteria for assignment of score points were uniform and consistent for new and published patients. A similar score for assessment of clinical severity of FOXG1 syndrome was introduced by Mencarelli et al. ${ }^{10}$ That score however implies some imbalance of the diverse domains of neurological involvement, e.g., impairment of speech development was rated with a maximum of 8 points, whereas epilepsy was rated with only 2 points.

The study was approved by the ethics committee of the Faculty of Medicine, University of Göttingen, Germany, and the relevant local institutional review boards. Parental (or legal guardian) written informed consent was obtained for all affected children.

\section{FOXG1 genotype groups}

FOXG1 variants in the new patients were identified during routine genetic testing using Sanger sequencing analysis in 10 patients from eight families or next-generation sequencing (NGS) technology in 20 patients from 20 families (NGS gene panel, whole-exome or trio-exome analysis) as indicated in Table $\mathbf{1 .}$

For statistical analysis of genotype-phenotype associations we divided the study patients (new and published cases) into five genetic subgroups according to the type and location of their variant within the following five specific FOXG1 domains: (i) N-terminal domain frameshift and nonsense variants $(n=37)$, (ii) forkhead domain conserved site 1 missense variants $(n=12)$, (iii) forkhead domain except conserved site 1 frameshift and nonsense variants $(n=9)$, (iv) forkhead domain except conserved site 1 missense variants $(n=9)$, and (v) C-terminal domain frameshift and nonsense variants $(n=9)$.

Seven patients were not assigned to one of these genotype groups for the following reasons:

Three patients carrying an in-frame variant (DB12-017a1 and DB12-017a2, G172M192del; ;,24 patient 20, P198del) and 


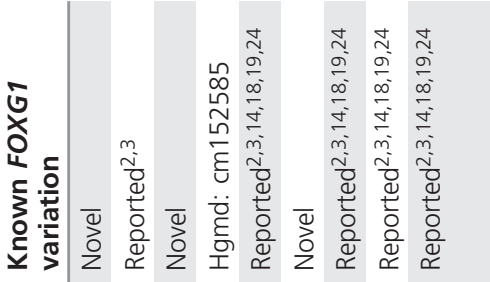

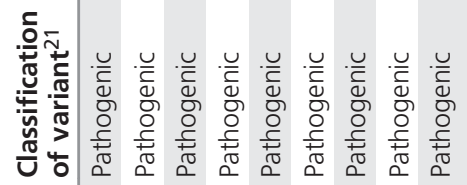

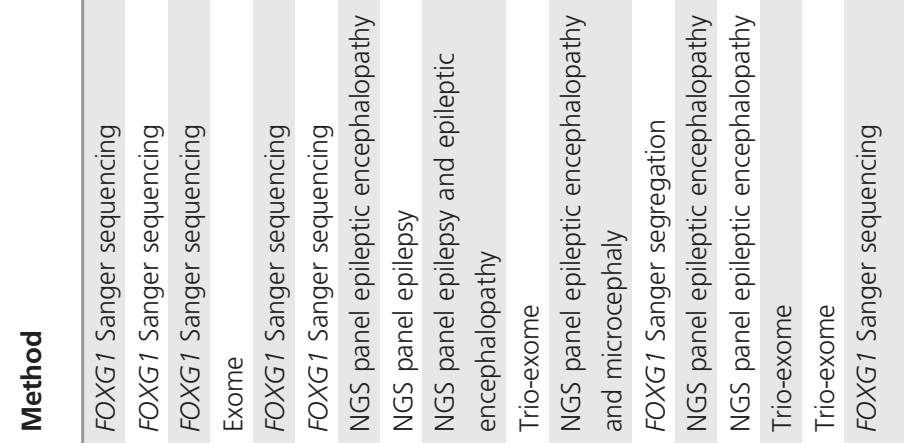

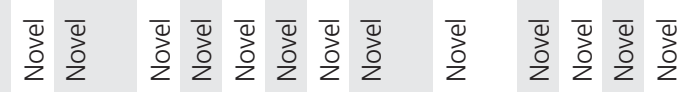
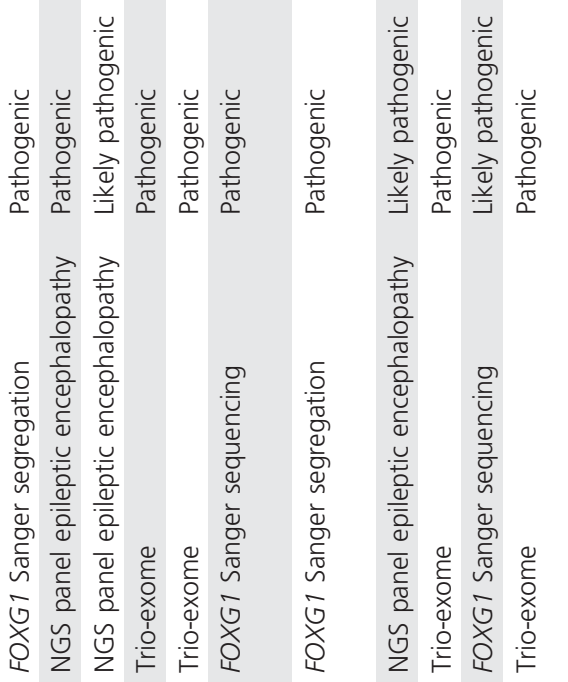

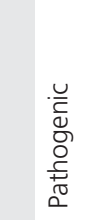
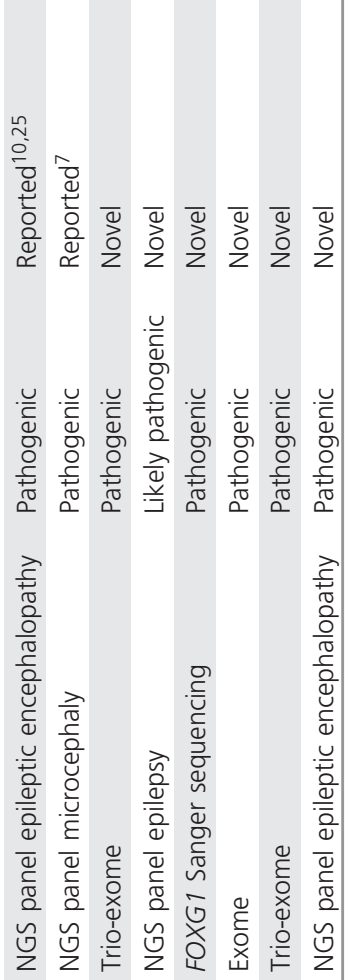

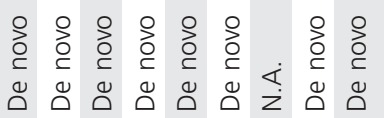

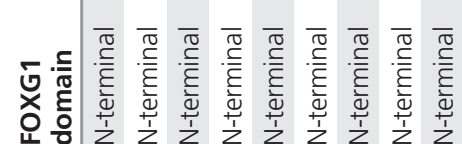

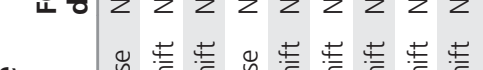

है

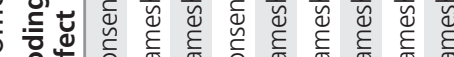

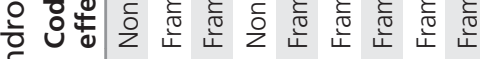

兵

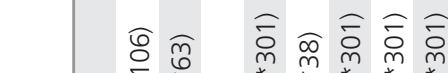

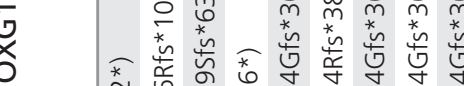

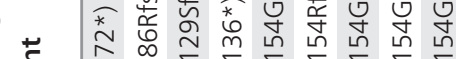

震

艺

要

3

.

氙

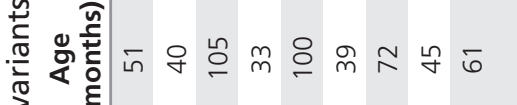

ज

인

宒号 o

文 $\sum_{0}^{0}$ वे

o c o co

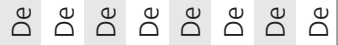

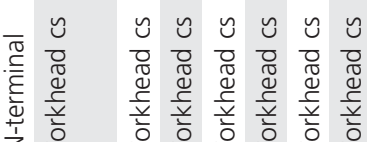

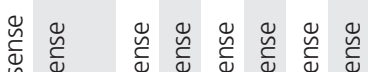

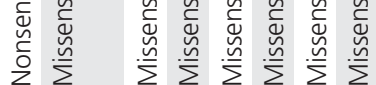

ธั จั จั จั จั

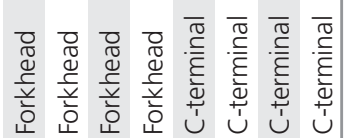

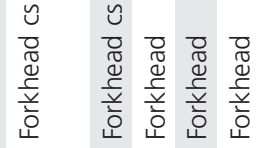

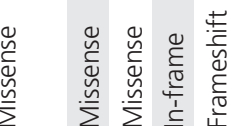

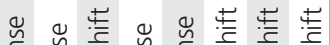

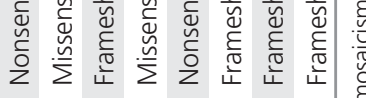

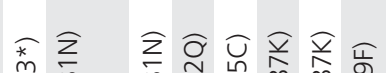

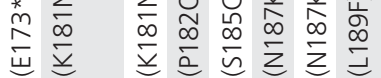

Q

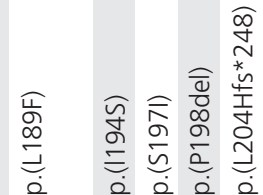

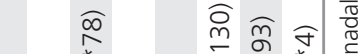

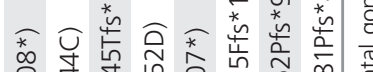

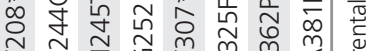

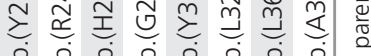

$-\cup \quad \cup \varangle r \varangle \varangle$

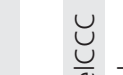

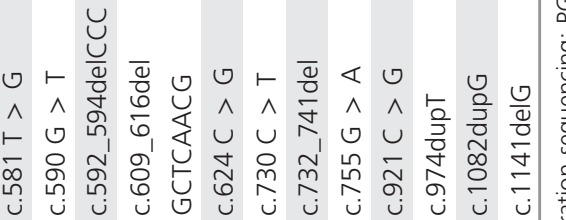

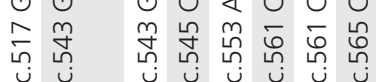

设

$\sim$ ๗

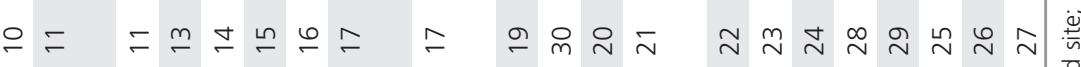

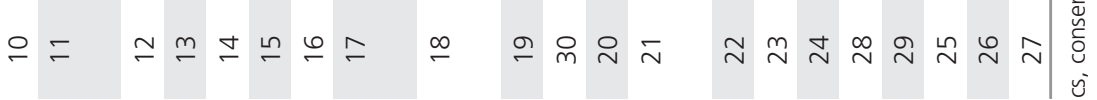


one patient carrying a frameshift variant in the forkhead domain conserved site 1 (RTT01158, S185Qfs*27010) were not assigned to one of the five genotype groups due to infrequent types and locations of the variants. Three patients carrying a missense variant at amino acid position 187 (N187K in patients 15 and 16, N187D in the patient reported by Terrone et al. ${ }^{26}$ ) showed particular clinical features markedly different from those observed in all other patients in genotype group 2 (see Results) and were therefore not included in this group. Fisher's exact test was used at the lowest level of test hierarchy (univariate tests of single measures), therefore a group of less than five patients was not expected to provide sufficient statistical power. Hence, while we used data for the whole cohort of 83 patients ( 30 new and 53 reported previously) for evaluating the clinical spectrum of FOXG1 syndrome (Table 2), only 76 patients (27 new, 49 published) were assigned to one of the five genotype groups and included in the statistical analysis of genotype-phenotype association (Table 3, Supplementary Table S3).

\section{Statistical analysis}

Statistical analysis was performed using $\mathrm{R}$, version 3.2.2 (http://cran.r-project.org). All $P$ values reported are twosided. Univariate association tests were performed with Fisher's exact test (all count data) or Kruskal-Wallis ranksum test (severity score, age). Multivariate tests were global tests of the five categories somatic growth, motor and speech development, behavior, neurological features, and MRI features (Table 3) and were performed by multivariate ranksum method ${ }^{27}$ (for details see Supplementary Material S5). The study had $80 \%$ power to detect differences between FOXG1 genotype groups regarding clinical severity for the presented design, sample sizes, data properties, and multipletesting adjustment.

\section{RESULTS}

The study comprised 83 patients from 76 families with a heterozygous FOXG1 variant, of whom 48 (58\%) were female and $35(42 \%)$ were male.

\section{Genotype analysis}

Among the 30 new patients (Table 1, Supplementary Table S1) we found 19 novel heterozygous FOXG1 variants not listed in the dbSNP, 1000 Genomes, and ExAC Browser databases (Figure 1a). Taking all new and previously reported patients together, we compiled 54 different heterozygous FOXG1 variants including 20 frameshift (37\%), 17 missense (31\%), 15 nonsense (28\%), and 2 in-frame variants $(4 \%)$ (Figure 1). All FOXG1 variants were classified according to $\mathrm{ACMG}^{21}$ as pathogenic (44 variants) or likely pathogenic (10 variants) (Supplementary Table S2). Variants are distributed over all FOXG1 protein domains, with 19 variants (35\%) in the $\mathrm{N}$-terminal domain, 10 variants $(18 \%)$ in the forkhead domain conserved site 1, 17 variants (31\%) in the remaining forkhead domain, and eight variants (15\%) in the C-terminal domain. This study confirms the previously reported two hotspots of frameshift variants in the N-terminal domain located at stretches of seven guanines and cytosines possibly prone to replication errors. ${ }^{1-3}$ Base pair 460 was affected in 16 patients: one patient with c.460delG (E154Rfs $\left.{ }^{\star} 38\right)$ and 15 patients from 13 families (17\% of all families) with c.460dupG (E154Gfs^301). Base pair 256 was altered in seven unrelated patients with c.256delC (Q86Rfs $\left.{ }^{\star} 106\right), \quad$ c.256dupC $\left(\mathrm{Q} 86 \mathrm{fs}^{\star} 34\right)$, or c.256 C > T $\left(\mathrm{Q} 86^{\star}\right)$. All other variants were single or double familial cases.

In the cohort of 83 patients, in 65 patients from 63 families de novo occurrence was demonstrated by parental testing. Siblings 17 and 18 are confirmed identical twins, the second pair of identical twins with a de novo FOXG1 variant reported to date. ${ }^{3}$ In eight patients from eight families, there were no data on segregation analysis in the parents. In one published family with three affected children, ${ }^{3,24}$ maternal somatic mosaicism was reported. In four additional families (7 patients) parental gonadal mosaicism was assumed. This group includes family 11 with two affected siblings (patients 11,12 ), and family 20 with a second affected sibling (clinical data for sibling not included in the study).

\section{Phenotype analysis}

Most of the 30 new patients showed clinical features identified previously as part of the core phenotype of FOXG1 syndrome. Table 2 compares clinical features in the whole cohort and in the 30 new and 53 published cases. Mean age at last follow-up was 105 months (range 14-384) for the whole cohort, 66 months (range 14-204) for new, and 128 months (range 21-384) for previously published patients. Compared to published cases, new patients were significantly younger $(P=0.0006)$, but also less often exhibited spasticity $(P=0.0007)$, tended to acquire functional hand use more often $(P=0.0076)$, and had lower severity scores $(P=0.0004$, score computable for 27 new and 28 published cases). All other phenotypic features were similar. Somatic growth was impaired in most patients. In the full cohort, microcephaly (head circumference $<-2$ SDS) was present in $24 \%$ at birth and in $84 \%$ at last follow-up. While $85 \%$ had normal length and $93 \%$ had normal weight at birth, $48 \%$ had short stature (length $<-2$ SDS) and 34\% were underweight (body mass index $<-2$ SDS) at follow-up.

Motor development was delayed in all patients and achievement of motor milestones (sitting, walking) had similar rates among the new and published cases. Unsupported sitting was achieved by $45 \%$ at a mean age of 28 months (range 5 to 108 months), unsupported walking by $15 \%$ at a mean age of 53 months (range 24 to 132 months). Functional hand use was observed in $40 \%$, but reported with a higher rate among new patients (60\%, borderline significance). Loss of motor skills was uncommon (18\%).

At last follow-up, 21\% showed some verbal expression. Age at first words was reported in 9 patients, with a mean age of 46 months (range 21 to 108 months). The mean number of spoken words was 19 (range 2 to 100 words) in those who spoke. 
Table 2 Phenotypic characterization of new and published patients with FOXG1 syndrome

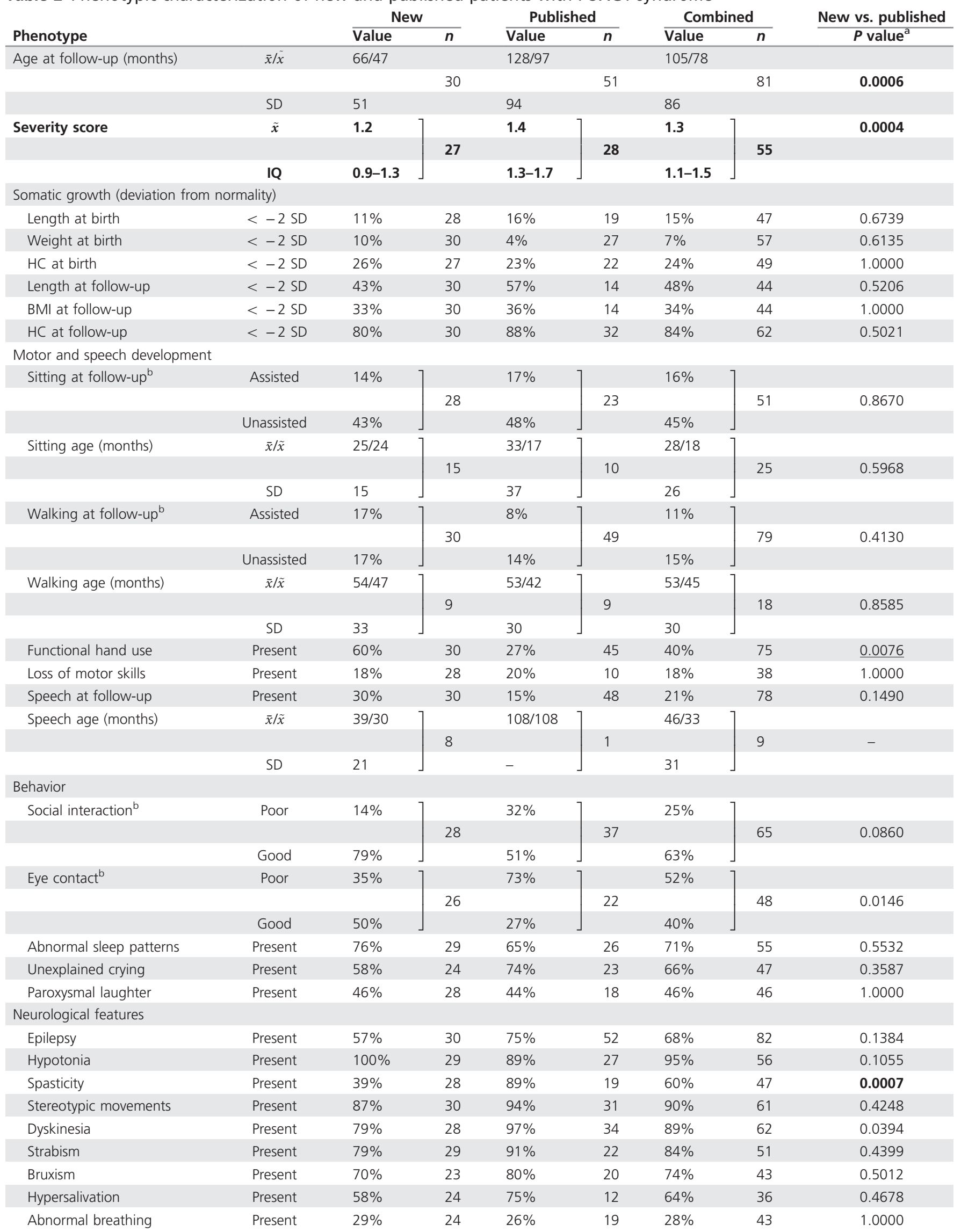


Table 2 Continued

\begin{tabular}{|c|c|c|c|c|c|c|c|c|}
\hline \multirow[b]{2}{*}{ Phenotype } & & \multicolumn{2}{|c|}{ New } & \multicolumn{2}{|c|}{ Published } & \multicolumn{2}{|c|}{ Combined } & \multirow{2}{*}{$\frac{\text { New vs. published }}{P \text { value }}$} \\
\hline & & Value & $n$ & Value & $n$ & Value & $n$ & \\
\hline \multicolumn{9}{|l|}{ Gastrointestinal features/others } \\
\hline Feeding difficulties & Present & $80 \%$ & 30 & $100 \%$ & 18 & $88 \%$ & 48 & 0.0708 \\
\hline Gastric reflux & Present & $52 \%$ & 27 & $84 \%$ & 19 & $65 \%$ & 46 & 0.0305 \\
\hline Constipation & Present & $69 \%$ & 29 & $84 \%$ & 19 & $75 \%$ & 48 & 0.3157 \\
\hline Kyphoscoliosis/scoliosis & Present & $28 \%$ & 25 & $55 \%$ & 20 & $40 \%$ & 45 & 0.1247 \\
\hline \multicolumn{9}{|l|}{ CMRI } \\
\hline Corpus callosum anomalies & Present & $56 \%$ & 27 & $77 \%$ & 30 & $67 \%$ & 57 & 0.1030 \\
\hline Delayed myelination & Present & $50 \%$ & 28 & $69 \%$ & 13 & $56 \%$ & 41 & 0.3210 \\
\hline Cortical anomalies & Present & $68 \%$ & 28 & $77 \%$ & 22 & $72 \%$ & 50 & 0.5374 \\
\hline
\end{tabular}

$\mathrm{BMI}$, body mass index; CMRI, cerebral magnetic resonance imaging; cS, conserved site; $\mathrm{HC}$, head circumference; IQ, interquartile range; $\bar{x}$, mean; $\tilde{x}$, median. ${ }^{a} \mathrm{New}$ and published data were compared by Fisher's exact test (categorical data) or Kruskal-Wallis rank-sum test (severity score and age variables; except speech age); significances are shown in bold (Bonferroni: $P \leq 0.05 / 36=0.0014)$, borderline significances are underlined. ${ }^{\mathrm{b}}$ Sitting, walking, social interaction, eye contact were rated in categories (unassisted/good, assisted/poor, present/absent); other variables in two categories (normal, pathological).

Hypotonia (95\%), stereotypic movements (90\%), dyskinesia (89\%), strabismus (84\%), bruxism (74\%), and spasticity $(60 \%)$ were prominent neurological features. However, spasticity was significantly less frequent among new (39\%) compared to published (89\%) patients.

Epilepsy was reported in $68 \%$ with a slightly higher rate ( $P=0.1384$, not significant $)$ among published cases as several patients had been ascertained from epilepsy focused studies. Mean age at onset of seizures, reported in 40 patients, was 25 months (range 3 to 168 months). The first quartile of these patients developed epilepsy by 8 months of age, median age at onset was 18 months, the third quartile developed epilepsy not before 29 months of age. Only one patient (W255*) reported by Ariani et al. ${ }^{9}$ had first epileptic seizures later than 6 years of age, at 168 months. A wide range of seizure types was observed including infantile spasms, focal, complex focal, generalized tonic, atonic and myoclonic seizures. Infantile spasms were reported in five patients in the study cohort of 83 cases $(6 \%)$, three new patients $\left(3, \mathrm{E} 136^{*} ; 15\right.$ and $\left.16, \mathrm{~N} 187 \mathrm{~K}\right)$ and two cases reported previously by Van der Aa et al. ${ }^{19}$ (case 1, A193T) and De Bruyn et al. ${ }^{22}$ (K170Qfs $\left.{ }^{\star} 285\right)$. The spasms were refractory to treatment.

Lennox-Gastaut syndrome was observed in the three unrelated patients with a missense variant affecting amino acid 187. In the two new patients (15 and 16) Lennox-Gastaut syndrome was preceded by infantile spasms starting at 4 months of age. The previously reported patient by Terrone et al. $^{26}$ (N187D) developed tonic seizures at 18 months that became drug resistant.

Evaluation of the photographs of the new patients revealed no specific facial features (pictures not shown).

\section{Neuroimaging}

Ages at cranial MRI ranged from 6 months to 16 years in the new patients. Neuroimaging features in the full cohort included mild to moderate hypoplasia and partial or complete aplasia of corpus callosum (67\%) as well as delayed myelination (56\%). Cortical anomalies included mild to moderate simplified gyral pattern and pachygyria (72\%).

\section{Genotype-phenotype association}

Table 3 displays probabilities of occurrences of clinical and neuroimaging features for the 76 patients (27 new, 49 published) with a FOXG1 variant assigned to one of the five genotype groups and highlights significant genotypephenotype associations. The FOXG1 severity score for global assessment of clinical and neuroimaging phenotypes revealed significantly higher severity in genotype group 1 compared to the other genotype groups 2 to 5 taken together $(P=0.0043)$ and in genotype group 1 compared to genotype group $2(P=0.0020$, Figure $1 \mathbf{b})$. Thus, carriers with a missense variant within the forkhead conserved site 1 presented with the mildest phenotype in the cohort. Severity was not significantly different between genotype groups 1 and $3(P=0.6620)$. However, variants of genotype group 4 $(P=0.1452)$ and $5(P=0.0437)$ tended to yield less severe phenotypes than genotype group 1 .

More differentiated insight was provided by analyses of associations between the five genotype groups and 29 phenotypic features (20 features included in the FOXG1 severity score plus nine additional features; see legend to Table 3, Supplementary Material S5). These features were assorted to five categories comprising somatic growth, motor and speech development, behavior, neurologic features, and cranial MRI anomalies. Multivariate testing of associations between FOXG1 genotype groups and phenotypic categories revealed consistent differences between genotype group 1 and genotype groups 2 to 5 taken together regarding motor and speech development $(P=0.0007)$, neurological features, and neuroradiological features $(P=0.0098$ and $P=0.0128$, borderline significant, Supplementary Figure S6) (Table 3, $P$-multivar). Of note, achievement of free sitting $(P=$ 0.00009), unsupported walking $(P=0.0001)$, and functional hand use $(P=0.0004)$ was significantly different between the five FOXG1 genotype groups and more 


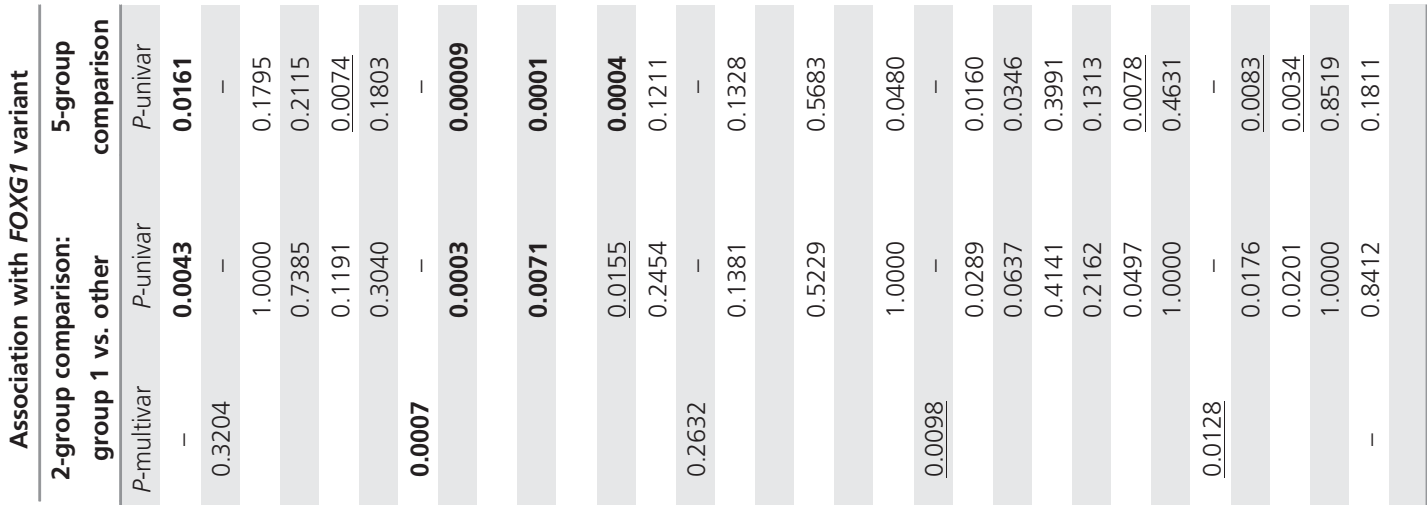

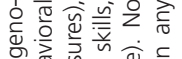

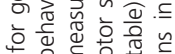

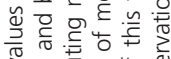

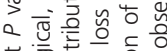

震 응 은.

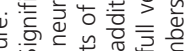

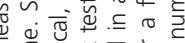

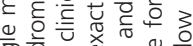

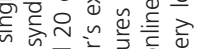

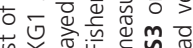

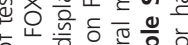

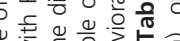

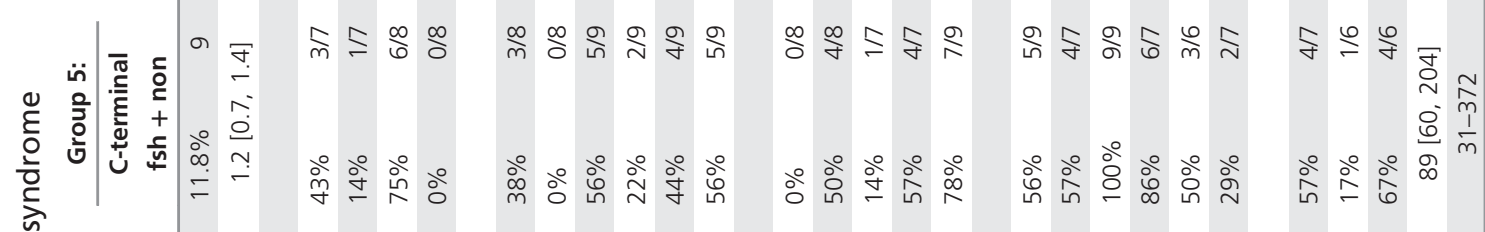

要

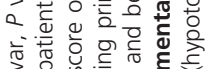

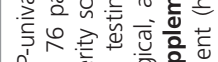

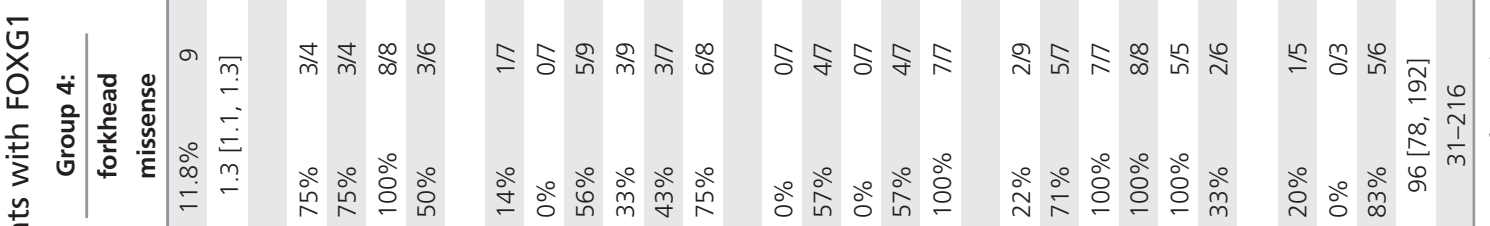

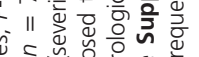

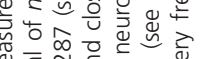

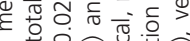

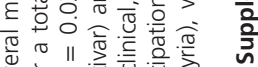

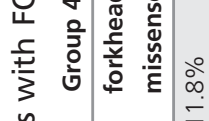

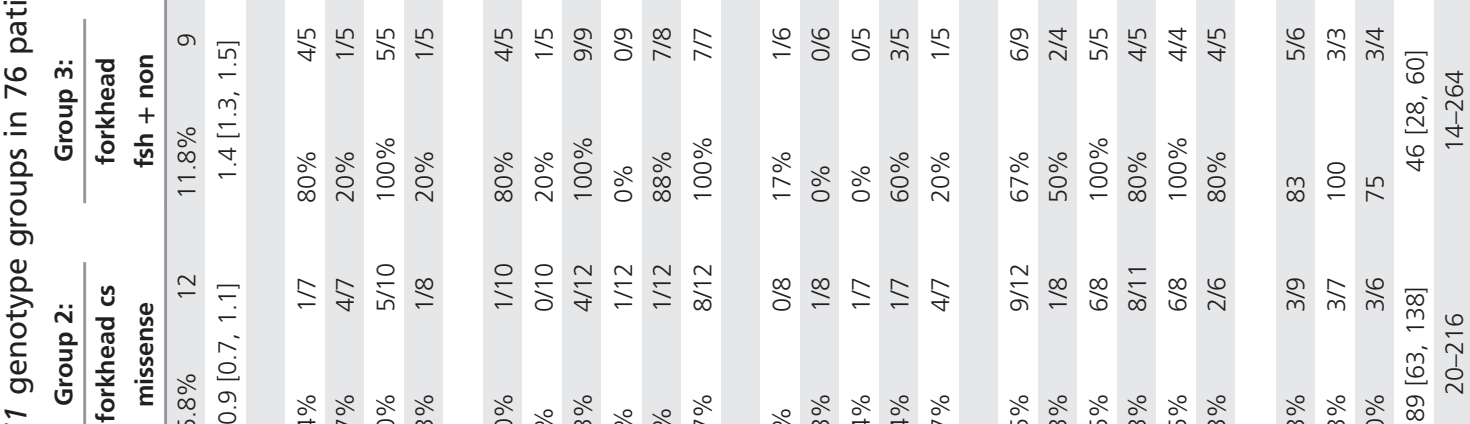

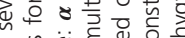

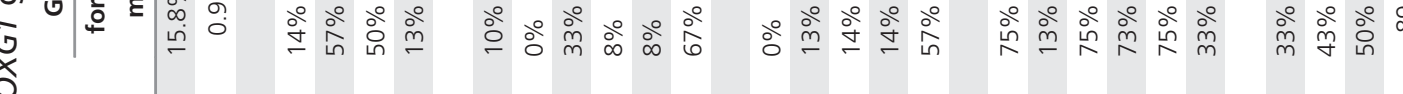

-

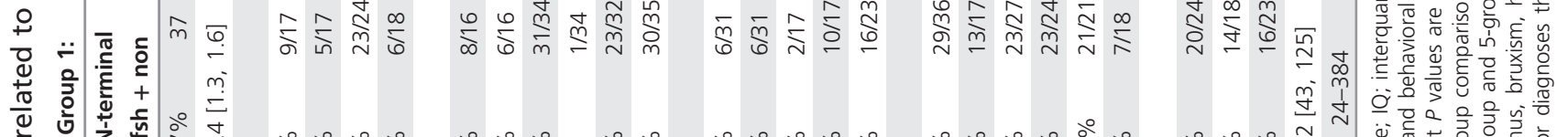

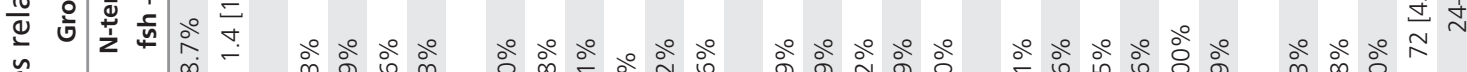

u

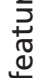

읃

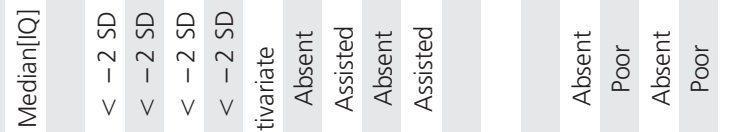
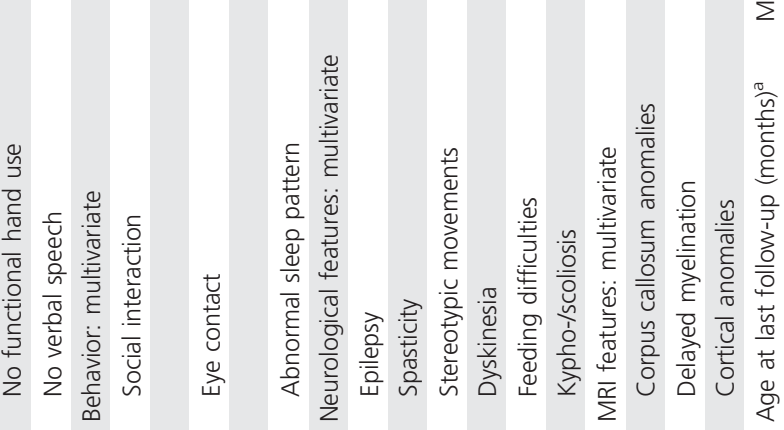

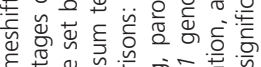

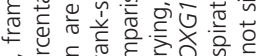

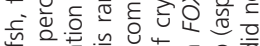

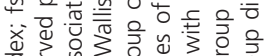

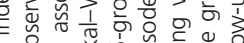

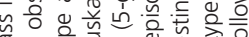

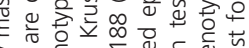

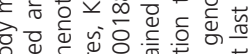

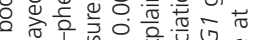

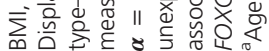



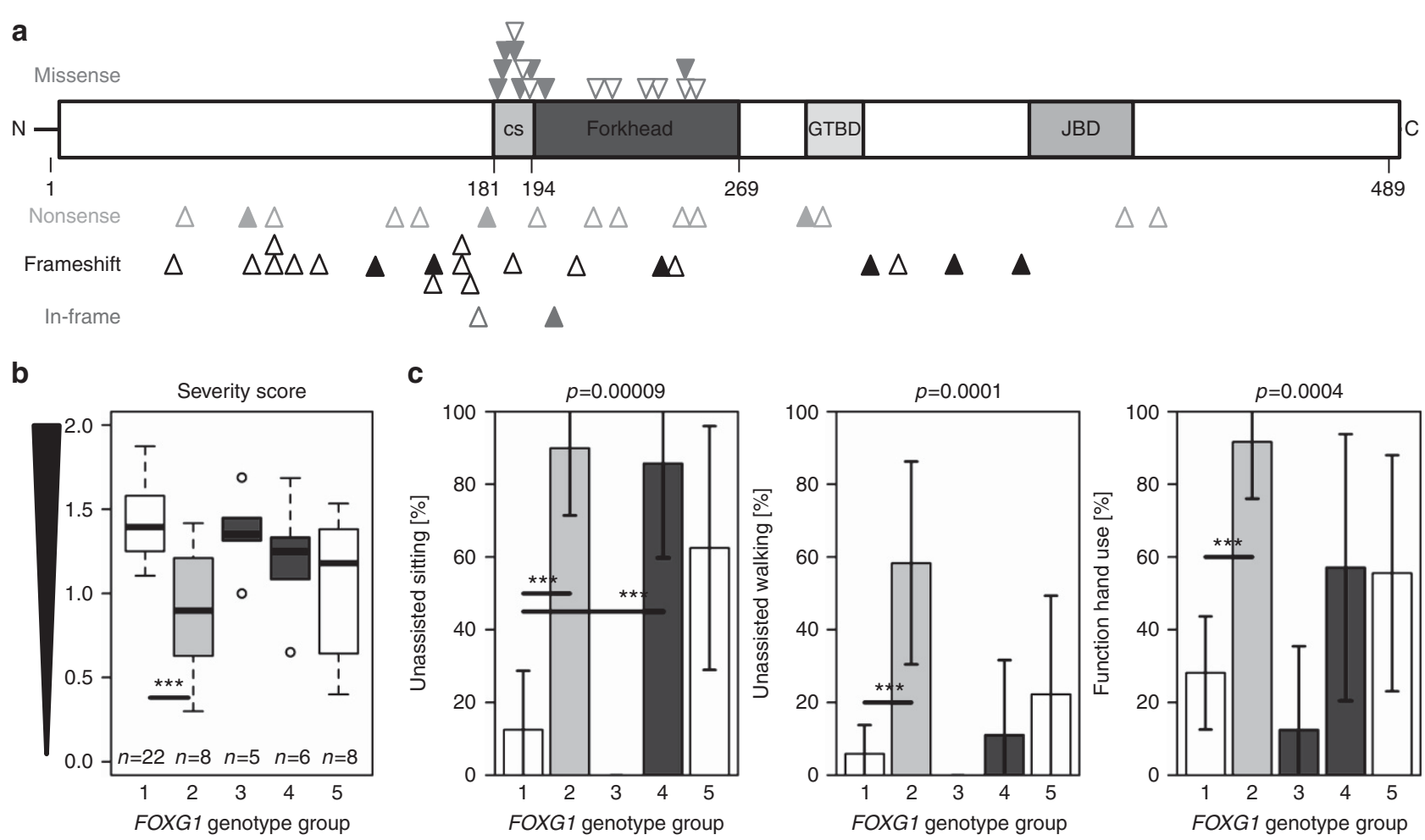
89

Figure 1 FOXG1 domains and distribution of variants. (a) Observed 54 FOXG1 variants in a schematic illustration; N-terminal domain, forkhead domain, forkhead domain conserved site 1 (cs), C-terminal domain of FOXG1 protein. Groucho-binding domain (GTBD, amino acids 307-406) and JARID1B-binding domain (JBD, amino acids 383-406) are indicated. Novel variants (full arrows), published variants (empty arrows). All missense variants (blue) cluster in the conserved forkhead domain including cs. Nonsense (green) and frameshift (black) variants are found in all protein domains. New and published FOXG1 variants are listed in Supplementary Table S2. Protein Ref Seq NP_005240.3. (b) Severity scores (boxplots) of the five FOXG1 genotype groups (group 1: N-terminal frameshift and nonsense variants; group 2: forkhead domain conserved site 1 missense variants; groups 3 and 4: forkhead domain except conserved site1, frameshift and nonsense variants (group 3) and missense variants (group 4); group 5: C-terminal frameshift and nonsense variants). Analysis revealed significantly lower severity (smaller score values) for carriers in genotype group 2 compared to group 1 $(* * * P=0.0020)$. (c) Motor ability (sitting, walking, functional hand use) differed significantly between FOXG1 genotype groups (***P $\leq 0.0017$ compared to group 1). Displayed are observed percentages of patients with $95 \%$ confidence intervals (error bars) who fully acquired these motor skills.

frequent in genotype group 2 compared to genotype group 1 (Figure 1c). Borderline significant differences between the five genotype groups were found concerning feeding difficulties, corpus callosum anomalies, delayed myelination (Supplementary Figure S6), and microcephaly at follow-up (less frequent in group 2, $P=0.0047$ compared to group 1, $P=0.0031$ compared with genotype groups $1,3,4$, and 5 taken together).

Analysis of the clinical phenotypes of all 83 patients showed that the three patients with an N187 variant showed markedly different clinical features compared with patients carrying other variants in the forkhead domain conserved site 1 . While the other patients assigned to genotype group 2 exhibit a relatively mild phenotype, the N187 patients are affected much more severely, including Lennox-Gastaut syndrome. This seems to constitute a special genotype-phenotype association, which would get lost by combining all cases with variants in the forkhead domain conserved site 1. It is known from other neurogenetic disorders that a very special missense variant may relate to a special clinical phenotype, as is the case, e.g., in cerebellar ataxia, areflexia, pes cavus, optic atrophy, and sensorineural hearing loss syndrome, which is associated with the c.2452G $>$ A (Glu818Lys) variant of the ATP1A3 gene and includes clinical features (optic atrophy, sensorineural hearing loss) not observed in patients with other ATP1A3 variants, even in close vicinity to position $818 .^{28}$

\section{DISCUSSION}

Evaluation of clinical, neuroimaging, and molecular data for this cohort of 30 new and 53 previously reported patients with FOXG1 syndrome/congenital variant of Rett syndrome associated with pathogenic and likely pathogenic variants in the forkhead box G1 gene indicates substantial variability in overall severity of the phenotype.

Discovery and initial delineation of the FOXG1 syndrome was related to the phenotypic similarities with Rett syndrome, and many patients described in the first years after original report of the FOXG1 syndrome were sequenced due to clinical resemblance of Rett syndrome. Now that NGS 
methods are more widely used in child neurology, indications for testing have been widened and many FOXG1 variants are detected in patients with a more unspecific clinical phenotype, including primary and secondary microcephaly, global developmental delay, and variable epilepsy types including infantile spasms and Lennox-Gastaut syndrome.

A recent study on epilepsy in FOXG1 syndrome found infantile spasms in six of seven patients with a FOXG1 duplication, but not in any of the children with deletions (4 cases) or intragenic FOXG1 variants (19 cases). ${ }^{3}$ Here, we report three new patients and two previously published cases with a FOXG1 variant and infantile spasms. ${ }^{19,22}$ Hence, infantile spams seem to be common in patients with a FOXG1 duplication, ${ }^{3}$ but are also part of the spectrum of epilepsy types seen in patients with a FOXG1 variant.

Our comparison of single clinical features between the 30 new and the 53 published cases reveals no striking phenotypic differences between these two groups. However, the FOXG1 severity score (accounting for clinical, behavioral, developmental, and MRI anomalies) introduced herein showed a significant difference between new and previously published patients with higher scores, which means overall more severe clinical features, in the previously reported patients (Table 2). The new patients were reported more often with functional hand use, showed higher rates of speech development, social interaction and eyecontact and were of younger age. This is plausible because of widened inclusion criteria for genetic analysis in younger patients. A further difference relates to the age of patients at diagnosis of the FOXG1 variant, as the new cases were clearly younger when the FOXG1 variant was detected, which is probably due to improved and more widely offered genetic testing.

While single cases of primary (congenital) microcephaly have been reported, ${ }^{2,18,26}$ microcephaly in FOXG1 syndrome has been presumed to be largely secondary (postnatal). Thus, the finding of primary microcephaly in $24 \%$ of the cohort reported herein is a new observation. However, analysis of the previous reports showed that in 59\% of the 53 published patients information on head circumference at birth was not provided; therefore, it was not possible to state whether microcephaly was congenital or postnatal in these cases.

Distinct genotype-phenotype associations could be delineated for five different FOXG1 genotype groups. The most severe phenotypes were found in patients with a FOXG1 frameshift or nonsense variant in the $\mathrm{N}$-terminal domain (genotype group 1) and the forkhead domain except conserved site 1 (genotype group 3). For example, most children in these groups did not learn unassisted sitting or walking and were not able to use their hands purposively. In comparison, significant milder phenotypes were associated with FOXG1 missense variants in the forkhead conserved site 1 (genotype group 2).

These genotype-phenotype associations are in accordance with the structure and function of the different FOXG1 protein domains. Truncating variants in the $\mathrm{N}$-terminal domain and the forkhead domain are predicted to result in a truncated protein with loss of the DNA binding forkhead domain, and correlate with the most severe phenotypes in the cohort (genotype groups 1 and 3). In contrast, truncating variants affecting the $\mathrm{C}$-terminal domain as well as missense variants in the forkhead domain may rather lead to a protein with residual function including preserved binding sites for corepressors such as Groucho binding domain and the JARID1B binding domain. Accordingly, these FOXG1 variants were found in children with milder phenotypes (genotype groups 2, 4, and 5). While truncation variants were detected in all FOXG1 domains, all missense variants cluster within the forkhead domain including conserved site 1. This region is particularly spared from genetic variation in healthy controls and shows the highest level of conservation among the FOXG1 domains.

FOXG1 is composed of one coding exon and belongs to the forkhead (FOX) family of genes identified in animals ranging from worm to human. ${ }^{29,30}$ FOXG1, a transcription repressor, is expressed in the fetal and adult brain. It is essential for the development of the forebrain (telencephalon) and for structures deriving from the telencephalon, including the cerebral cortex, hippocampus, and basal ganglia in mice. ${ }^{31}$ FOXG1 affects the early phase of cortical development by regulating progenitor cell proliferation and differentiation in the neocortex and is considered a key promoter of neocortical lamination. ${ }^{32}$ Recent research indicated a critical role for Foxg1 in the formation of the postnatal and adult hippocampal dentate gyrus ${ }^{33}$ and in interneuron development. ${ }^{34}$ These functional characteristics of FOXG1 do not explain the full clinical phenotype, but may relate to single, though unspecific features such as intellectual disability, dystonichyperkinetic movement disorder, and dysplasia of predominantly the frontal part of the corpus callosum.

It is widely assumed that recurrence risk is low in a fully penetrant severe autosomal dominant disease. Yet, highly variable frequencies of germline mosaicism in autosomal dominant disorders have been reported. In Dravet syndrome, up to $7 \%$ germline mosaicism have been found. ${ }^{35}$ In NIPBLrelated Cornelia de Lange syndrome, recurrence risks to sibs of unaffected parents are estimated with $1.5 \%{ }^{36}$ In our cohort, $5 \%$ of the families $(4 / 76)$ had more than one affected child with a FOXG1 variant (excluding identical twins). In only one of three previously described families with several affected children, ${ }^{3,24}$ maternal somatic mosaicism for the FOXG1 likely pathogenic variant was documented. ${ }^{24}$ The parents of the patients in these families were reported as unaffected. Therefore, in genetic counseling of parents of a patient with an apparent de novo variant, gonadal mosaicism needs to be considered. Prenatal genetic testing should be offered in all pregnancies.

Clinical application of whole-exome analysis in patients with intellectual disability or complex neurological diseases including epilepsy recently revealed additional cases with a FOXG1 pathogenic variant ${ }^{37,38}$ indicating that the frequency of FOXG1 syndrome may have been underestimated. The variable frequencies of a wide spectrum of FOXG1 associated clinical features, the nonspecific facial features, and the 
variable presence of brain anomalies make clinical diagnosis of FOXG1 syndrome difficult, especially in very young patients. Overlapping phenotypes are seen in patients with other neurodevelopmental disorders, including Rett syndrome, Angelman syndrome, and CDKL5-, ARX-, and STXBP1-related encephalopathies. Our observations indicate that besides chromosomal rearrangements Angelman syndrome and Rett syndrome were the main differential diagnoses excluded by previous genetic testing (Supplementary Table S1). This is in line with the designation of the FOXG1 syndrome as congenital variant of Rett syndrome, and for a clinician with broad experience with Rett syndrome and its variants the FOXG1 associated phenotype may be clinically recognizable.

FOXG1 syndrome/congenital variant of Rett syndrome will likely be diagnosed more frequently in the future due to the wide and increasing application of NGS technologies. NGS panels of genes associated with microcephaly, epilepsy, or epileptic encephalopathy should include FOXG1. Collection of larger numbers of patients will allow for further delineation of the phenotypic variability in FOXG1 syndrome.

\section{SUPPLEMENTARY MATERIAL}

Supplementary material is linked to the online version of the paper at http://www.nature.com/gim

\section{ACKNOWLEDGMENTS}

The study was supported by a grant from the Niedersächsische Ministerium für Wissenschaft und Kultur (Ministry of Science and Culture of Lower Saxony, Germany), file 74ZN1284. The sponsor of the study had no role in study design, data collection, data analysis, data interpretation, or writing of the report. This study would not have been possible without the invaluable assistance of the patients and their families. We thank the cooperating physicians for referral of the patients.

\section{DISCLOSURE}

K.L.H. is a full-time employee of Ambry Genetics. I.P. and K. Hoertnagel are employed by and receive a salary from CeGaT $\mathrm{GmbH}$. FOXG1 testing and exome sequencing are among the commercially available products of both institutions. The other authors declare no conflict of interest.

\section{REFERENCES}

1. Allou L, Lambert L, Amsallem D, et al. $14 q 12$ and severe Rett-like phenotypes: new clinical insights and physical mapping of FOXG1regulatory elements. Eur J Hum Genet. 2012;20:1216-1223.

2. Kortüm $F$, Das $S$, Flindt $M$, et al. The core FOXG1 syndrome phenotype consists of postnatal microcephaly, severe mental retardation, absent language, dyskinesia, and corpus callosum hypogenesis. J Med Genet. 2011:48:396-406.

3. Seltzer LE, Ma M, Ahmed S, et al. Epilepsy and outcome in FOXG1related disorders. Epilepsia 2014;55:1292-1300.

4. Bisgaard $A-M$, Kirchhoff $M$, Tümer $Z$, et al. Additional chromosomal abnormalities in patients with a previously detected abnormal karyotype, mental retardation, and dysmorphic features. Am J Med Genet A. 2006;140:2180-2187.

5. Jacob FD, Ramaswamy V, Andersen J, Bolduc FV. Atypical Rett syndrome with selective FOXG1 deletion detected by comparative genomic hybridization: case report and review of literature. Eur J Hum Genet. 2009;17:1577-1581.

6. Kumakura A, Takahashi S, Okajima K, Hata D. A haploinsufficiency of FOXG1 identified in a boy with congenital variant of Rett syndrome. Brain Dev. 2014;36:725-729.

7. Le Guen T, Fichou Y, Nectoux J, et al. A missense mutation within the fork-head domain of the forkhead box G1 Gene (FOXG1) affects its nuclear localization. Hum Mutat 2011;32:35.

8. Le Guen T, Bahi-Buisson N, Nectoux J, et al. A FOXG1 mutation in a boy with congenital variant of Rett syndrome. Neurogenetics 2011;12: $1-8$.

9. Ariani F, Hayek $G$, Rondinella $D$, et al. FOXG1 is responsible for the congenital variant of Rett syndrome. Am J Hum Genet. 2008;83:89-93.

10. Mencarelli MA, Spanhol-Rosseto A, Artuso R, et al. Novel FOXG1 mutations associated with the congenital variant of Rett syndrome. J Med Genet. 2010;47:49-53.

11. Mencarelli MA, Kleefstra T, Katzaki E, et al. $14 q 12$ Microdeletion syndrome and congenital variant of Rett syndrome. Eur J Med Genet. 2009;52:148-152.

12. Papa FT, Mencarelli MA, Caselli R, et al. A $3 \mathrm{Mb}$ deletion in $14 q 12$ causes severe mental retardation, mild facial dysmorphisms and Rett-like features. Am J Med Genet. 2008:146A:1994-1998.

13. Shoichet SA, Kunde S-A, Viertel $P$, et al. Haploinsufficiency of novel FOXG1B variants in a patient with severe mental retardation, brain malformations and microcephaly. Hum Genet 2005;117:536-544.

14. Cellini E, Vignoli A, Pisano T, et al. The hyperkinetic movement disorder of FOXG1-related epileptic-dyskinetic encephalopathy. Dev Med Child Neurol. 2016;58:93-97.

15. Santen GWE, Sun Y, Gijsbers ACJ, et al. Further delineation of the phenotype of chromosome 14q13 deletions: (positional) involvement of FOXG1 appears the main determinant of phenotype severity, with no evidence for a holoprosencephaly locus. J Med Genet. 2012;49:366-372.

16. Yeung $A$, Bruno $D$, Scheffer IE, et al. $4.45 \mathrm{Mb}$ microduplication in chromosome band $14 q 12$ including FOXG1 in a girl with refractory epilepsy and intellectual impairment. Eur J Med Genet. 2009;52: 440-442.

17. Brunetti-Pierri N, Paciorkowski AR, Ciccone R, et al. Duplications of FOXG1 in 14q12 are associated with developmental epilepsy, mental retardation, and severe speech impairment. Eur J Hum Genet. 2010;19: 102-107.

18. Bahi-Buisson N, Nectoux J, Girard B, et al. Revisiting the phenotype associated with FOXG1 mutations: two novel cases of congenital Rett variant. Neurogenetics 2010;11:241-249.

19. van $\operatorname{der} A a N$, van den Bergh $M$, Ponomarenko $N$, Verstraete $L$, Ceulemans B, Storm K. Analysis of FOXG1 is highly recommended in male and female patients with Rett syndrome. Mol Syndromol. 2011;1: 290-293.

20. Philippe C, Amsallem D, Francannet $C$, et al. Phenotypic variability in Rett syndrome associated with FOXG1 mutations in females. I Med Genet. 2010;47:59-65

21. Richards S, Aziz N, Bale $S$, et al. Standards and guidelines for the interpretation of sequence variants: a joint consensus recommendation of the American College of Medical Genetics and Genomics and the Association for Molecular Pathology. Genet Med 2015;17:405-424.

22. Bruyn $C$ de, Vanderhasselt $T$, Tanyalçin I, et al. Thin genu of the corpus callosum points to mutation in FOXG1 in a child with acquired microcephaly, trigonocephaly, and intellectual developmental disorder: a case report and review of literature. Eur J Paediatr Neurol. 2014;18: 420-426.

23. Filippis $R$ de, Pancrazi $L$, Bjørgo $K$, et al. Expanding the phenotype associated with FOXG1 mutations and in vivo FoxG1 chromatin-binding dynamics. Clin Genet 2012;82:395-403.

24. McMahon KQ, Papandreou A, Ma M, et al. Familial recurrences of FOXG1-related disorder: evidence for mosaicism. Am J Med Genet A. 2015;167:3096-3102.

25. Roche-Martínez A, Gerotina E, Armstrong-Morón J, Sans-Capdevila Ó, Pineda M. FOXG1, a new gene responsible for the congenital form of Rett syndrome. Rev Neurol 2011:52:597-602.

26. Terrone G, Bienvenu T, Germanaud D, et al. A case of Lennox-Gastaut syndrome in a patient with FOXG1-related disorder. Epilepsia 2014:55:9.

27. Malzahn D, Schillert A, Müller M, Bickeböller H. The longitudinal nonparametric test as a new tool to explore gene-gene and gene-time effects in cohorts. Genet Epidemiol 2010;34:469-478. 
28. Sweney MT, Newcomb TM, Swoboda KJ. The expanding spectrum of neurological phenotypes in children with ATP1A3 mutations, alternating hemiplegia of childhood, rapid-onset dystonia-Parkinsonism, CAPOS and beyond. Pediatr Neurol 2015;52:56-64.

29. Bredenkamp N, Seoighe C, Illing N. Comparative evolutionary analysis of the FoxG1 transcription factor from diverse vertebrates identifies conserved recognition sites for microRNA regulation. Dev Genes Evol. 2007;217:227-233.

30. Wiese $S$, Murphy DB, Schlung A, et al. The genes for human brain factor 1 and 2, members of the fork head gene family, are clustered on chromosome 14q. Biochim Biophys Acta. 1995;1262:105-112.

31. Dou C-L. Dual role of brain factor-1 in regulating growth and patterning of the cerebral hemispheres. Cereb Cortex 1999;9:543-550.

32. Hanashima C, Shen L, Li SC, Lai E. Brain factor-1 controls the proliferation and differentiation of neocortical progenitor cells through independent mechanisms. J Neurosci. 2002;22:6526-6536.
33. Tian C, Gong Y, Yang Y, et al. Foxg1 has an essential role in postnatal development of the dentate gyrus. J Neurosci 2012;32:2931-2949.

34. Yang $Y$, Shen $W$, Ni $Y$, Su Y, Yang Z, Zhao C. Impaired interneuron development after Foxg1 disruption. Cereb Cortex 2017;27: 793-808.

35. Depienne C, Arzimanoglou A, Trouillard O, et al. Parental mosaicism can cause recurrent transmission of SCN1A mutations associated with severe myoclonic epilepsy of infancy. Hum Mutat 2006;27:389.

36. Jackson $L$, Kline $A D$, Barr MA, Koch $S$. de Lange syndrome: a clinical review of 310 individuals. Am J Med Genet. 1993;47:940-946.

37. Helbig KL, Farwell Hagman KD, Shinde DN, et al. Diagnostic exome sequencing provides a molecular diagnosis for a significant proportion of patients with epilepsy. Genet Med 2016;18:898-905.

38. Retterer K, Juusola J, Cho MT, et al. Clinical application of wholeexome sequencing across clinical indications. Genet Med 2016;18: 696-704. 